\title{
Functional Gametes Derived from Explants of Single Blastomeres Containing the "Germ Plasm" in Xenopus laevis: A Genetic Marker Study
}

\author{
KOHJI IKENISHI \\ Department of Biology, Faculty of Science, Osaka City University, Osaka 558, Japan; and Hubrecht Laboratory, \\ Netherlands Institute for Developmental Biology, Utrecht, The Netherlands \\ Received November 18, 1986; accepted in revised form February 6, 1987
}

\begin{abstract}
Single blastomeres containing the "germ plasm" were isolated from 32-cell embryos of Xenopus albino $\left(a^{p} / a^{p}\right)$ or wild type and cultured in vitro until the corresponding normal control embryos reached the neurula stage. The resulting explants from albinos were implanted into wild-type host neurulae and vice versa. The formation of functional gametes, eggs or sperm, of donor type was tested when the operated host embryos had reached sexual maturity. The color of the eggs laid by the experimental females and the presence or absence of melanophores in the epidermis and of pigment granules in the eyes of hatched larvae from matings of the experimental males with albino females made possible the identification of donor-type gametes. Twelve males and 12 females of the wild-type hosts, and 16 males and 14 females of the albino hosts survived. Six animals produced donor-type eggs or sperm, most of them being germ line chimeras. This shows that functional gametes can develop from explants derived from single blastomeres containing the "germ plasm." co 1987 Academic Press, Inc.
\end{abstract}

\section{INTRODUCTION}

It is well known that in normal development of anuran amphibians primordial germ cells (PGCs) in the embryonic gonad at tadpole stages originate from the cells containing the "germ plasm" at cleavage stages (for review, see Nieuwkoop and Sutasurya, 1979). In a previous study (Ikenishi et al., 1984) we demonstrated with radioisotope labeling that PGCs developed from explants of single blastomeres containing the "germ plasm," isolated from Xenopus stage- 6 embryos, when the explants were implanted into host neurulae. This implies that, as far as the differentiation of the PGCs is concerned, the developmental process progressed normally in the isolated blastomeres, notwithstanding the long period of in vitro culture (ca. $21 \mathrm{hr}$ at $22-24^{\circ} \mathrm{C}$ ). Recently it was also shown in the same experimental system that PGCs could originate from single somatic blastomeres into which vegetal pole cytoplasm or a subcellular fraction of it (the germ cell determinant) was microinjected (Ikenishi el al., 1986).

Blackler and Fischberg (1961) and Blackler (1962) grafted the endodermal region of neurulae between species or different subspecies of Xenopus and showed that functional gametes of graft origin were produced in the host animals; these gametes therefore originated from the presumptive PGCs (pPGCs) in the endodermal cell mass of the graft [pPGCs are those PGCs that have not yet reached the embryonic gonad (Whitington and Dixon, 1975)]. In our studies (Ikenishi et al., 1984, 1986), however, there is no proof that PGCs produced experimentally from hlastomeres containing the "germ plasm," or from somatic blastomeres by means of microinjection, can develop into functional gametes in host animals. In Drosophila it is reported that experimentally induced PGCs in the embryonic gonad can never differentiate into functional gametes in the adult fly (Okada and Togashi, 1985).

In order to test whether PGCs originating from explants can differentiate into functional gametes, in the present study grafting experiments between albino and wild-type embryos of Xenopus are performed. Some of the experimental animals are demonstrated to produce donor-type gametes.

\section{MATERIALS AND METHODS}

Fertilized Xenopus eggs of both albino $\left[a^{p} / a^{p}\right.$, periodic albinism (Hoperskaya, 1975)] and wild type were obtained by injecting sexually mature males and females with gonadotropic hormone, as described earlier (Kotani et al., 1973). Eggs were dejellied with sodium thioglycollate solution as described previously (Ikenishi, 1982). Embryos were staged after Nieuwkoop and Faber (1967). An outline of the present experiment is shown in Fig. 1 . The isolation and culture of single blastomeres containing the "germ plasm" from 32-cell embryos (stage 6) of both genotypes were carried out in the same manner as in the previous study (Ikenishi et al., 1984), except that the culture medium contained no isotope. The ex- 

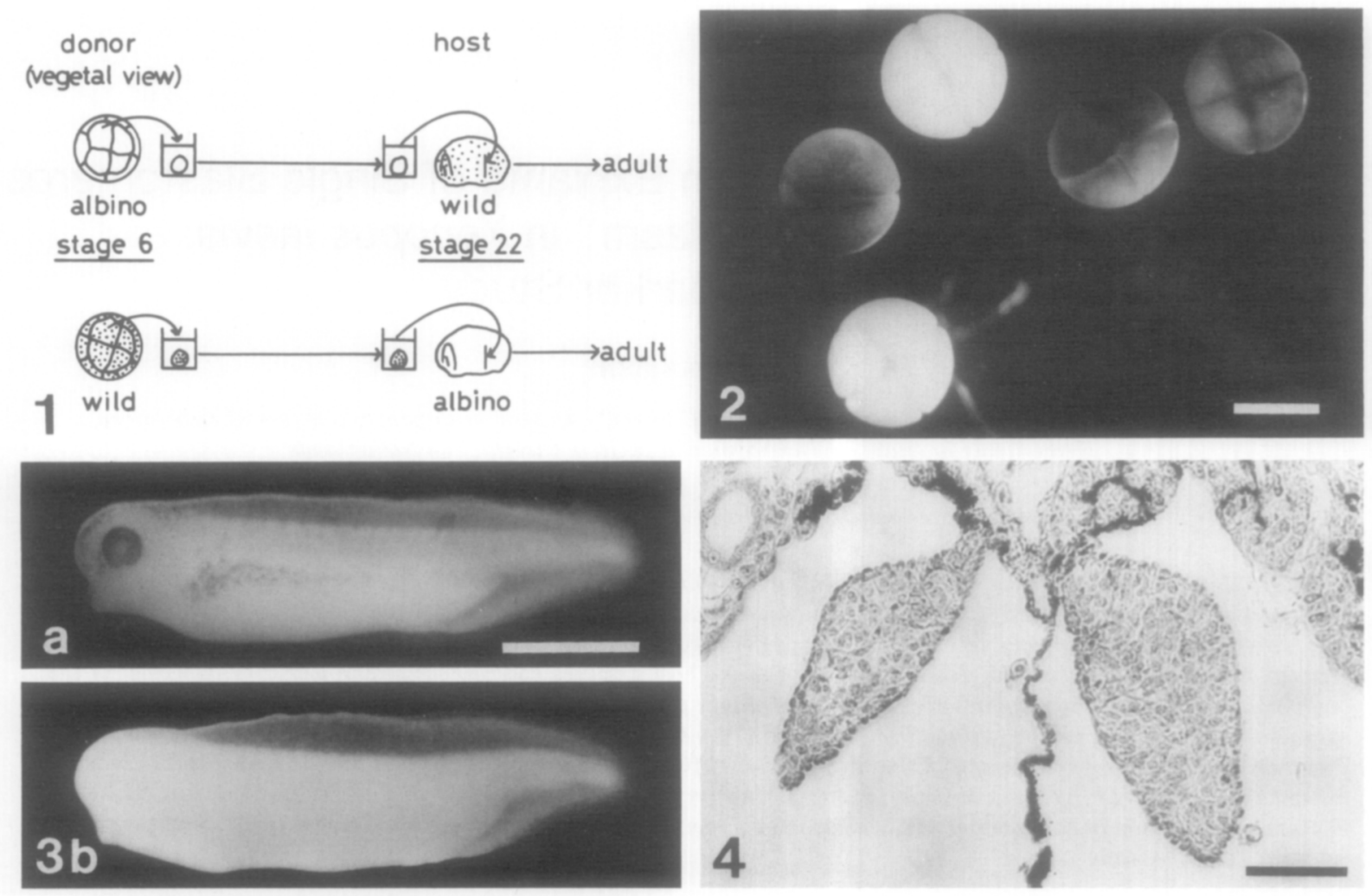

FIG. 1. Schematic representation of the grafting experiment between albino and wild type in Xenopus. Single blastomeres containing the "germ plasm" were isolated from the most vegetal tier of 32-cell embryos of Xenomes albino $\left(a^{p} / a^{p}\right)$ or wild type and cultured until the corresponding normal control embryos had reached the neurula stage. The resulting explants from albino donors were implanted into the posterior part of host wild-type neurulae, and vice versa. After wound healing the operated embryos were allowed to develop to the adult stage.

FIG. 2. Two- and four-cell embryos of both albino and wild type. They developed from eggs laid by a wild-type experimental female, which was a germ line chimera, and a wild-type male (see text). Bar: $1 \mathrm{~mm}$.

FIG. 3. Hatched larvae (stage 33/34) from the mating of an experimental albino male, which was a germ line chimera, and an albino female (male gamete identification test, see text). (a) Larva with melanophores in the skin and pigment granules in the eyes, which is externally indistinguishable from a wild-type larvae $(+/+)$; in this case the sperm must have been of donor type $(+)$. (b) Larva without melanophores and pigment granules; in this case the sperm must have been of host type $\left(a^{p}\right)$. Bar: $1 \mathrm{~mm}$.

FIG. 4. The testes of a larva (stage 55) developed from a wild-type egg laid by an experimental albino female, fertilized with sperm (Z) of a normal male. All the offspring of this female were males (ZZ), which means that the grafted male pPGCs were sex-reversed in the host ovaries. Bar: $50 \mu \mathrm{m}$.

plants from albino were implanted into the posterior part of wild-type host neurulae (stage 22), and vice versa, according to the methods used in the previous study (Ikenishi et al., 1984). After wound healing had occurred the operated embryos were kept in 1/10 Holtfreter's solution until the tadpole stage. They were then transferred to dechlorinated tap water and reared to sexual maturity.

In order to know whether or not the prospective sex of grafted pPGCs, which is determined by the sex genotype, can be reversed in hosts of the opposite sex, the gonads of the larvae developed from the donor-type eggs laid by one specific experimental albino female (see Results) were fixed in Bouin's fixative overnight, dehydrated in an ethanol series, and embedded in paraplast. Sections ( $8 \mu \mathrm{m}$ in thickness) were stained with Mayer's hematoxylin and eosin and examined to determine the gonadal sex.

Identification of donor-type gametes in host animals. In the experimental females identification was possible by recording the color of the eggs laid. Wild-type and albino eggs are easily distinguishable by their color; in the former the animal hemisphere is dark brown, while in the latter both hemispheres are milky white. In the experimental males identification was indirect. They were mated with albino females and the presence or $a b-$ sence of melanophores in the epidermis and of pigment granules in the eyes of hatched larvae (stage 33/34) from these matings was recorded. If the fertilizing sperm is wild-type, the resultant heterozygotes $\left(a^{p} /+\right)$ will have melanophores and pigment granules at hatching because wild type is dominant in melanophore differentiation 
over albino, $\left(a^{p} / a^{p}\right)$ being a homozygous recessive (Hoperskaya, 1975). If the fertilizing sperm is albino, the resultant homozygotes $\left(a^{p} / a^{p}\right)$ will never have melanophores and pigment granules.

\section{RESULTS AND DISCUSSION}

Of the wild-type hosts, 12 males and 12 females survived and reached sexual maturity (Table 1 ). Of the albino hosts 16 males and 14 females developed to the adult stage. Three of the 24 wild-type hosts and 3 of the 30 albino hosts have produced donor-type gametes so far (Table 1). Five out of these six animals producing donor-type gametes were germ line chimeras. Three wild-type females and one albino produced both types of fertilizable eggs when mated with a male (Fig. 2). This is in good agreement with the results obtained in Xenopus by Blackler (1962) showing that donor-type gametes are able to retain their characteristics notwithstanding a passage through host gonads of a different subspecies. One albino male produced both types of hatched larvae (pigmented and nonpigmented) in the gamete identification test (see Methods) (Fig. 3). The remaining albino female laid only wild-type eggs.

It has thus been proved that functional gametes can originate from the blastomeres of 32-cell embryos containing the "germ plasm" upon isolation and culture in vitro for ca. $21 \mathrm{hr}$. This implies that the isolation and culture conditions in the present study do not disturb the process of differentiation into functional gametes.

In the present grafting experiments, combinations between embryos of unlike sex genotypes were probably made. In such cases the question arises as to whether or not the prospective sex of the gafted pPGCs, which is determined by their sex genotype, can be reversed in the host gonads. For the analysis of such sex-reversal, significant numbers of donor-type gametes are required. Fortunately, the one albino female already mentioned

TABLE 1

Number of Experimental Animals Producing DONOR-TYPE GAMETES

\begin{tabular}{lllllc}
\hline Host & $\begin{array}{c}\text { Number of animals } \\
\text { surviving from } \\
\text { operated host } \\
\text { embryos }\end{array}$ & & \multicolumn{2}{c}{$\begin{array}{c}\text { Number of animals } \\
\text { producing donor- } \\
\text { type gametes }\end{array}$} \\
\cline { 2 - 3 } \cline { 5 - 6 } Wild-type & 12 & & & Male & Female \\
Albino & 16 & 12 & & 0 & 3 \\
\hline
\end{tabular}

\footnotetext{
a Three wild-type females, one albino male, and one of the two albino females produced both donor- and host-type gametes, while the remaining albino female laid only donor-type eggs.
}

exclusively laid donor-type (wild-type) eggs in significant numbers. Histological examination of the gonads of 15 stage-55 larvae derived from these wild-type eggs, fertilized with normal sperm [the sex genotype of which is $\mathrm{Z}$ (Gallien, 1955, 1956)], showed that all the larvae were males (ZZ) (Fig. 4). In other words, the sex genotype of all the eggs was $\mathrm{Z}$. This indicates that the sex genotype of the grafted pPGCs which produced the eggs was ZZ, instead of $\mathrm{ZW}$ as in pPGCs of normal females. This means that the grafted male pPGCs were sex-reversed in the host ovaries, as also reported by Blackler (1965).

All the chimeric females of the present study produced many fewer donor-type than host-type eggs. This may be explained in part as follows. Usually all four blastomeres around the vegetal pole of stage- 6 embryos contain "germ plasm" (Dixon, 1981; Ikenishi, 1982). In the present study only one of those blastomeres was isolated, whereas the host neurulae developed from stage- 6 embryos with four blastomeres containing the plasm. The number of blastomeres containing the plasm in the blastula, i.e., the founder clone for the germ line cells, is thought to determine the number of the PGCs formed at the tadpole stage (Dixon, 1981). If the proportion of donor-type oogonia in host animals is assumed to correspond to that of donor-type PGCs at the tadpole stage, theoretically the percentage of donor-type oogonia in the host animals should be only 20 .

In our previous study (Ikenishi et al., 1984) the percentage of tadpoles containing PGCs of graft origin was ca. 45 . In the present study the percentage of adult animals producing donor-type gametes was ca. 11 (Table 1). This difference does not necessarily mean that not all tadpoles having donor-type PGCs reach the adult stage and produce donor-type gametes. Rather, it is possible that the nonproducers of donor-type gametes, which are at most 2 years old now, may still produce gametes from the grafted $\mathrm{pPGCs}$ later on, since not all the oogonia or spermatogonia in the gonads become eggs or sperm at the same time.

The present results clearly show that the pPGCs in the explants derived from single blastomeres containing the "germ plasm" differentiated into functional gametes in the hosts. Therefore, it is likely that the labeled PGCs in the previous study (Ikenishi et al., 1984), which was carried out in essentially the same manner, could also continue the developmental pathway to functional gametes. Recently we demonstrated that PGCs can develop from explants derived from single somatic blastomeres into which the vegetal pole cytoplasm or a subcellular fraction of it was injected (Ikenishi et al., 1986). By using the same genetic marker as in the present experiments it may be possible to find out whether PGCs originating from microinjected somatic blastomeres can differentiate in to functional gametes. 
I am grateful to Drs. S. W. de Laat and J. Faber, Hubrecht Laboratory, Utrecht, The Netherlands, for giving me the opportunity to carry out the grafting experiments in that laboratory. I also thank Dr. M. Kotani, Department of Natural Science, Osaka Women's University, and Dr. M. Furusawa, Deputy Director of Research Institute, Daiichi Seiyaku Co. Ltd., for valuable advice and critical reading of the manuscript, and to Dr. J. Faber for critical reading and correction of the English. Thanks are expressed to Ms. R. Verhoeff-de Fremery and Mr. F. J. M. Vervoordeldonk of the Hubrecht Laboratory for taking care of my experimental animals, and to Mr. Y. Tsuzaki in my laboratory for the care and breeding of the experimental animals and the histological examination of the larval gonads. The costs of my stay in the Netherlands were covered in part by a maintenance grant from the Hubrecht Laboratory.

\section{REFERENCES}

BLACKLER, A. W. (1962). Transfer of primordial germ cells between two subspecies of Xenopus laevis. J. Embryol. Exp. Morphol. 10, 641651.

BLACKLER, A. W. (1965). Germ-cell transfer and sex ratio in Xenopus laevis. J. Embryol. Exp. Morphol. 13, 51-61.

Blackler, A. W., and FischBeRG, M. (1961). Transfer of primordial germ-cells in Xenopus laevis. J. Embryol. Exp. Morphol. 9, 634-641.

Dixon, K. E. (1981). The origin of the primordial germ cells in the amphibia. Neth. J. Zool. 31, 5-37.

Gallien, L. (1955). Descendance unisexuée d'une femelle de Xenopus laevis Daud. ayant subi pendant sa phase larvaire l'action gynogène du benzoate d'oestradiol. C. R. Acad. Sci. Paris 240, 913-915.
GalliEN, L. (1956). Inversion expérimentale du sexe chez un anoure inférieur, Xenopus laevis D. Analyse des conséquences génétiques. Bull. Biol. 90, 163-181.

HoperskayA, O. A. (1975). The development of animals homozygous for a mutation causing periodic albinism $\left(a^{p}\right)$ in Xenopus laevis. $J$. Embryol. Exp. Morphol. 34, 253-264.

IKENISHI, K. (1982). A possibility of an in vitro differentiation of primordial germ cells (PGCs) from blastomeres containing "germinal plasm" of early cleavage stage in Xenopus laevis. Dev. Growth Different. 24, 205-215.

IKENISHI, K., Nakazato, S., and OKUDA, T. (1986). Direct evidence for the presence of germ cell determinant in vegetal pole cytoplasm of Xenopus laevis and in a subcellular fraction of it. Dev. Growth Different. 28, 585-590.

Ikenishi, K., Okuda, T., and Nakazato, S. (1984). Differentiation of presumptive primordial germ cell (pPGC)-like cells in explants into PGCs in experimental tadpoles. Dev. Biol. 103, 258-262.

Kotani, M., Ikenishi, K., and Tanabe, K. (1973). Cortical granules remaining after fertilization in Xenopus laevis. Dev. Biol. 30, 228232.

NieuwKoop, P. D., and Faber, J. (1967). "Normal Table of Xenopus laevis (Daud)," 2nd ed. North-Holland, Amsterdam.

Nieuwkoop, P. D., and Sutasurya, L. A. (1979). "Primordial Germ Cells in the Chordates." Cambridge Univ. Press, Cambridge.

OKADA, M., and ToGaSHI, S. (1985). Isolation of a factor inducing pole cell formation from Drosophila embryos. Int. J. Invertebr. Reprod. Dev. 8, 207-217.

Whitington, P. MCD., and Dixon, K. E. (1975). Quantitative studies of germ cells during early embryogenesis of Xenopus laevis. J. Embryol. Exp. Morphol. 33, 57-74. 